OPEN ACCESS

Check for updates FAST TRACK
For numbered affiliations see end of the article.

Correspondence to: V C Veiga viviane.veiga@bp.org.br (ORCID 0000-0002-0287-3601)

Additional material is published online only. To view please visit the journal online.

Cite this as: $B M / 2021 ; 372: n 84$ http://dx.doi.org/10.1136/bmj.n84

Accepted: 11 January 2021

\section{Effect of tocilizumab on clinical outcomes at 15 days in patients with severe or critical coronavirus disease 2019: randomised controlled trial}

\author{
Viviane C Veiga, ${ }^{1,2}$ João A G G Prats, ${ }^{1}$ Danielle L C Farias, ${ }^{1}$ Regis G Rosa, ${ }^{2,3}$ Leticia K Dourado, \\ Fernando G Zampieri,, ${ }^{2,4}$ Flávia R Machado, ${ }^{2,5}$ Renato D Lopes, ${ }^{6,7}$ Otavio Berwanger, ${ }^{8}$ \\ Luciano C P Azevedo, ${ }^{2,9}$ Álvaro Avezum, ${ }^{10}$ Thiago C Lisboa, ${ }^{2,4}$ Salomón S O Rojas, ${ }^{1}$ \\ Juliana C Coelho, ${ }^{1}$ Rodrigo T Leite, ${ }^{1}$ Júlio C Carvalho, ${ }^{1}$ Luis E C Andrade, ${ }^{11}$ Alex F Sandes, ${ }^{11}$ \\ Maria C T Pintão, ${ }^{11}$ Claudio G Castro Jr, ${ }^{8,12}$ Sueli V Santos, ${ }^{4}$ Thiago M L de Almeida, ${ }^{5}$ \\ André N Costa, ${ }^{9}$ Otávio C E Gebara, ${ }^{13}$ Flávio G Rezende de Freitas, ${ }^{2,14}$ Eduardo S Pacheco, ${ }^{14}$ \\ David J B Machado, ${ }^{15}$ Josiane Martin, ${ }^{15}$ Fábio G Conceição, ${ }^{15}$ Suellen R R Siqueira, ${ }^{15}$ \\ Lucas P Damiani, ${ }^{4,16}$ Luciana M Ishihara, ${ }^{16}$ Daniel Schneider, ${ }^{3}$ Denise de Souza, ${ }^{3}$ \\ Alexandre B Cavalcanti, ${ }^{2,4}$ Phillip Scheinberg ${ }^{1}$; on behalf of the Coalition covid-19 Brazil VI \\ Investigators
}

\section{ABSTRACT}

OBJECTIVE

To determine whether tocilizumab improves clinical outcomes for patients with severe or critical coronavirus disease 2019 (covid-19).

\section{DESIGN}

Randomised, open label trial.

SETTING

Nine hospitals in Brazil, 8 May to 17 July 2020.

\section{PARTICIPANTS}

Adults with confirmed covid-19 who were receiving supplemental oxygen or mechanical ventilation and had abnormal levels of at least two serum biomarkers (C reactive protein, $\mathrm{D}$ dimer, lactate dehydrogenase, or ferritin). The data monitoring committee recommended stopping the trial early, after 129 patients had been enrolled, because of an increased number of deaths at 15 days in the tocilizumab group. INTERVENTIONS

Tocilizumab (single intravenous infusion of $8 \mathrm{mg} / \mathrm{kg}$ ) plus standard care $(n=65)$ versus standard care alone $(n=64)$.

\section{MAIN OUTCOME MEASURE}

The primary outcome, clinical status measured at 15 days using a seven level ordinal scale, was analysed as a composite of death or mechanical ventilation because the assumption of odds proportionality was not met.

\section{WHAT IS ALREADY KNOWN ON THIS TOPIC}

In coronavirus disease 2019 (covid-19), an increased level of interleukin 6 correlates with disease severity and mortality

The interleukin 6 inhibitor tocilizumab might mitigate the inflammatory response and improve clinical outcomes of patients with severe or critical covid-19

The effects on clinical outcomes are, however, not well defined

\section{WHAT THIS STUDY ADDS}

Among patients with severe or critical covid-19, tocilizumab plus standard care was not superior to standard care alone in improving clinical status at 15 days, and it might increase mortality

\section{RESULTS}

A total of 129 patients were enrolled (mean age 57 (SD 14) years; 68\% men) and all completed followup. All patients in the tocilizumab group and two in the standard care group received tocilizumab. 18 of $65(28 \%)$ patients in the tocilizumab group and 13 of $64(20 \%)$ in the standard care group were receiving mechanical ventilation or died at day 15 (odds ratio $1.54,95 \%$ confidence interval 0.66 to $3.66 ; \mathrm{P}=0.32$ ). Death at 15 days occurred in 11 (17\%) patients in the tocilizumab group compared with $2(3 \%)$ in the standard care group (odds ratio 6.42, 95\% confidence interval 1.59 to 43.2). Adverse events were reported in 29 of $67(43 \%)$ patients who received tocilizumab and

\section{CONCLUSIONS}

In patients with severe or critical covid-19, tocilizumab plus standard care was not superior to standard care alone in improving clinical outcomes at 15 days, and it might increase mortality.

\section{TRIAL REGISTRATION}

ClinicalTrials.gov NCT04403685.

\section{Introduction}

The coronavirus disease 2019 (covid-19) pandemic has led to profound worldwide health, economic, and social losses. ${ }^{1-3}$ As of October 2020, more than 40 million people have received a diagnosis of covid-19 and one million deaths have occurred globally. ${ }^{1}$ Although the disease is asymptomatic or mild in most patients, a substantial percentage of people have more extensive pneumonia that can progress to hypoxaemic respiratory failure, shock, dysfunction of organs, and death. ${ }^{4}$ Activation of macrophages as a result of infection, initially in the lungs and then systemically, is an essential source of pro-inflammatory cytokines and chemokines. ${ }^{56}$ This host immune response is thought to play a key role in the pathophysiology of lung and other organ dysfunction in covid-19. ${ }^{78}$

Tocilizumab is an interleukin 6 inhibitor approved for the treatment of rheumatoid arthritis, giant cell arteritis, and cytokine release syndrome during 21 of 62 (34\%) who did not receive tocilizumab. 
chimeric antigen receptor $\mathrm{T}$ cell therapy (CAR-T). ${ }^{9}$ Interleukin 6 is an inflammatory cytokine that exerts its effects in the liver and on lymphocytes, inducing acute phase reactants such as $\mathrm{C}$ reactive protein, fibrinogen, and hepcidin from hepatocytes, and promotes CD4 T helper 17 and CD8 cytotoxic T cell differentiation and antibody production. ${ }^{10}$ Interleukin 6 plays an important role in controlling viral infections such as influenza A, severe acute respiratory syndrome coronavirus 1 , and herpesvirus. ${ }^{11}$ In covid-19, an increased level of interleukin 6 and $C$ reactive protein correlates with disease severity and mortality. ${ }^{12} 13$ Thus, blocking interleukin 6 activity might play a role in mitigating the inflammatory response and improve clinical outcomes in patients with covid-19. To test this hypothesis, we conducted a randomised controlled trial comparing tocilizumab plus standard care with standard care alone in patients admitted to hospital with severe or critical covid-19.

\section{Methods}

This multicentre, randomised, open label, parallel group, superiority trial was conducted in nine hospitals across Brazil. The trial protocol and statistical analysis plan were submitted for publication before interim analysis (see supplementary file). ${ }^{14}$ Written or electronic consent was obtained from all patients or legal representatives before study enrolment. The trial was overseen by an independent data monitoring committee. Because of an administrative error by the research team, the trial was registered at ClinicalTrials.gov a few days after enrolment of the first patients (see supplementary file). An independent adjudication committee analysed secondary infections and deaths. Details of the trial rationale and methods have been described elsewhere and are provided in the study protocol. ${ }^{14}$

\section{Patients}

We enrolled hospital in-patients aged 18 years or older with severe acute respiratory syndrome coronavirus 2 (SARS-CoV-2) infection, confirmed by reverse transcription-polymerase chain reaction, and with symptoms for more than three days. Eligible patients had severe or critical covid-19, ${ }^{15}$ with evidence of pulmonary infiltrates confirmed by chest computed tomography or radiography, and were receiving supplemental oxygen to maintain oxygen saturation greater than $93 \%$ or had been receiving mechanical ventilation for less than 24 hours before analysis. In addition, at least two of the following criteria had to be met: D dimer $>2.74 \mathrm{nmol} / \mathrm{L}(>1000 \mathrm{ng} / \mathrm{mL})$, C reactive protein $>50 \mathrm{mg} / \mathrm{L}(>5 \mathrm{mg} / \mathrm{dL})$, ferritin $>300 \mu \mathrm{g} / \mathrm{L}$, or lactate dehydrogenase greater than the upper limit of normal. Exclusion criteria included active uncontrolled infection, raised aspartate aminotransferase or alanine aminotransferase levels greater than five times the upper limit of normal, and renal disease with an estimated glomerular filtration of $<30 \mathrm{~mL} / \mathrm{min} / 1.72 \mathrm{~m}^{2}$. See the supplementary file for full details of the inclusion and exclusion criteria.

\section{Trial procedures}

Patients were randomised in a 1:1 ratio to receive either standard care or tocilizumab plus standard care, with random blocks of sizes 2, 4, 6, and 8, and stratified by age ( $<60$ and $\geq 60$ years) and sex, according to a computer generated schedule using the sample function of software R 3.6.3 (R Foundation). Allocation concealment was ensured by a central automated web accessed system (REDCap), developed by CZO. The concomitant use of hydroxychloroquine, azithromycin, corticosteroids, and antibiotics was allowed according to standard care per local institutional guidelines for patients with covid-19. Remdesivir was not available in Brazil. In the experimental group, tocilizumab was administered as a single intravenous infusion at a dose of $8 \mathrm{mg} / \mathrm{kg}$ (maximum $800 \mathrm{mg}$ ).

Data were collected daily, from randomisation until day 29, in the electronic case report forms. Hospital researchers, unblinded to treatment assignment, collected outcome data during the patients' hospital stay. For patients who were discharged before day 15, an interviewer who was unaware of the assigned trial group conducted a structured telephone call with the patient or patient's proxy on or after day 15 to assess vital status and return to routine activities.

\section{Outcomes}

The primary outcome was clinical status at 15 days evaluated with the use of a seven level ordinal scale, defined as: level 1-not admitted to hospital and with no limitation in activities, level 2-not admitted to hospital but with limitation in activities, level 3admitted to hospital and not receiving supplemental oxygen, level 4-admitted to hospital and receiving supplemental oxygen, level 5-admitted to hospital and receiving non-invasive positive pressure ventilation or high flow oxygen through a nasal cannula, level 6-admitted to hospital and receiving mechanical ventilation, and level 7-death.

Secondary outcomes were all cause mortality, ascertained from data analysed to day 28; in-hospital mortality; sequential organ failure assessment score at eight and 15 days; clinical status at eight days, assessed using a six level ordinal scale (see supplementary file), and at 29 days, assessed using a seven level ordinal scale; ventilator-free days within 29 days; time to independence from supplemental oxygen within 29 days; duration of hospital stay; secondary infections; occurrence of thromboembolic events; and adverse events. Other prespecified exploratory outcomes were levels of serum inflammatory markers and cytokines, measured at days 5 and 8 (see supplementary file).

\section{Statistical analysis}

We estimated that an initial sample size of 150 patients would provide $80 \%$ power to detect an odds ratio of 0.44 of having a higher seven level ordinal scale at 15 days, with a two sided significance level of $5 \%$. The primary analysis followed the intention-to-treat principle, except for adverse events, which were analysed in a safety population that included patients according to 
the drug received, regardless of assigned group. The primary outcome was initially planned to be assessed with ordinal logistic regression assuming proportional odds ratios adjusted for stratification variables (age and sex). Because the assumption of odds proportionality was not met (Brant test $\mathrm{P}=0.04$ ), we collapsed the seven level ordinal scale into a binary outcome (levels 1 to 5 (alive and not receiving mechanical ventilation) versus levels 6 and 7 (receiving mechanical ventilation or death)) and used logistic regression for assessment, as specified in the statistical analysis plan. The effect on the primary outcome represents the odds ratio for receiving mechanical ventilation or death (levels 6 and 7) versus better clinical levels at day 15 .

A sensitivity analysis of the treatment effect on the primary outcome was performed using a per protocol population and considering only patients who received the treatment as assigned. We further conducted a post hoc sensitivity analysis of the effect on the primary outcome adjusting for baseline clinical status on the seven level ordinal scale, owing to imbalance in the baseline distribution of this variable between treatment groups, plus age and sex. Analyses for prespecified subgroups were conducted with interaction terms (see supplementary file).

Secondary outcomes were evaluated by generalised linear regression using appropriate distributions. To account for the competing risks of death we performed a post hoc analysis assessing the treatment effect on duration of hospital admission including only patients who were discharged alive. All models were adjusted for age, as a continuous variable, and sex. Results are presented with corresponding 95\% confidence intervals. One interim analysis was planned when 75 patients had completed 15 days of follow-up. An independent data monitoring committee performed the interim analysis.

Analysis was performed with $\mathrm{R}$ software ( $\mathrm{R}$ Core Team). ${ }^{16} \mathrm{P}$ values are not reported for secondary or exploratory outcomes. The widths of the confidence intervals for secondary and exploratory outcomes were not adjusted for multiple comparisons, thus the intervals should not be used to infer definitive treatment effects.

\section{Patient and public involvement}

No patients were involved in setting the research question, or in developing plans for recruitment, design, implementation, and dissemination of the results of this study. The study was conceived under the covid-19 pandemic.

\section{Results}

A total of 129 patients were recruited between 8 May and 17 July 2020. The trial was prematurely interrupted on 17 July 2020, after the first interim analysis, in accordance with the recommendation of the data monitoring committee, owing to an excess number of deaths at 15 days in the tocilizumab group (see supplementary file). Because of the rapid rate of enrolment, when complete data for 15 day follow- up was available for the first 75 patients and the first interim analysis was conducted, 129 patients had been enrolled in the trial (first patient enrolled on 8 May 2020). Follow-up for the last patient was completed on 11 August 2020. Table 1 in the supplementary file presents the number of patients enrolled according to site. Sixty five patients were assigned to receive tocilizumab plus standard care, and all were treated accordingly (fig 1). Sixty four patients were assigned to receive standard care alone; however, two patients received tocilizumab at the discretion of the treating doctors. The 15 day follow-up was completed for all patients.

Table 1 and supplementary table S2 present the baseline characteristics of the included patients. Mean age was 57 (SD 14) years and 68\% of patients were men (table 1). Hypertension, diabetes, and obesity were the most common comorbidities. Seven per cent of patients were receiving corticosteroids at enrolment. The use of other drugs (antibiotics, antivirals, and corticosteroids) did not differ between the groups in the first 15 days (supplementary table S3). More patients in the tocilizumab group were using supplementary oxygen at enrolment $(60 \% v 44 \%)$, whereas use of non-invasive ventilation or high flow oxygen through a nasal cannula was higher in the control group (23\% $v 41 \%)$.

\section{Primary outcome}

Table 2 and supplementary figure S1 show the distribution of the primary outcome (seven level ordinal scale at 15 days) and figure 2 shows patient status over time according to study group. Supplementary table S4 presents the cumulative proportions of the categories on the seven level ordinal scale and shows that the odds proportionality assumption did not hold. The use of tocilizumab was not associated with an improvement in mechanical ventilation or death at 15 days (18 of $65(28 \%)$ patients in the tocilizumab group and 13 of $64(20 \%)$ in the standard care group: odds ratio $1.54,95 \%$ confidence interval 0.66 to 3.66; $\mathrm{P}=0.32$ ). Death at 15 days, a component of the primary outcome, occurred in $11(17 \%)$ patients in the tocilizumab plus standard care group compared with two (3\%) in the standard care group (odds ratio 6.42, 1.59 to 43.2). A prespecified per protocol sensitivity analysis and a post hoc sensitivity analysis adjusted for baseline clinical status on the seven level ordinal scale were also not indicative of treatment benefit (see supplementary file). The effects on the primary outcome were in general similar across prespecified subgroups (supplementary table S6).

\section{Secondary outcomes}

Tocilizumab was not associated with either detectable significant differences on mortality up to 28 days (odds ratio $2.70,95 \%$ confidence interval 0.97 to 8.35 ) or in-hospital mortality (2.70, 0.97 to 8.35) (table 2). Supplementary tables S7 and S8 show the causes of death. Clinical status at day 8 and day 29 was not significantly different between treatment groups. 


\begin{tabular}{|c|c|c|}
\hline Characteristics & Tocilizumab group $(n=65)$ & Control group $(n=64)$ \\
\hline Mean (SD) age (years) & $57.4(15.7)$ & $57.5(13.5)$ \\
\hline Men & $44(68)$ & $44(69)$ \\
\hline Mean (SD) days from symptom onset to randomisation & $10.0(3.1)$ & $9.5(3.0)$ \\
\hline \multicolumn{3}{|l|}{ Comorbidities: } \\
\hline Hypertension & $30(46)$ & $34(53)$ \\
\hline Diabetes & $22(34)$ & $20(31)$ \\
\hline Obesity & $15(23)$ & $16(25)$ \\
\hline Heart failure & $4(6)$ & $3(5)$ \\
\hline Myocardial infarction & $4(6)$ & $3(5)$ \\
\hline Chronic obstructive pulmonary disease & $2(3)$ & $2(3)$ \\
\hline Asthma & $4(6)$ & $1(2)$ \\
\hline Chronic kidney disease & $5(8)$ & $1(2)$ \\
\hline Solid malignancy & $4(6)$ & $5(8)$ \\
\hline Haematological malignancy & $1(1)$ & $0(0)$ \\
\hline \multicolumn{3}{|l|}{ Previous drug use: } \\
\hline None & $13(20)$ & $9(14)$ \\
\hline Corticosteroid ( $>5 \mathrm{mg}$ prednisone for $>30$ days) & $4(6)$ & $5(8)$ \\
\hline Other immunosuppressants* & $5(5)$ & $2(3)$ \\
\hline Hydroxychloroquine & $11(17)$ & $9(14)$ \\
\hline Azithromycin & $23(35)$ & $31(48)$ \\
\hline Otherst & $41(63)$ & $38(59)$ \\
\hline \multicolumn{3}{|l|}{ Vasopressor: } \\
\hline None & $56(86)$ & $57(89)$ \\
\hline \multicolumn{3}{|l|}{ Norepinephrine $(\mu \mathrm{g} / \mathrm{kg} / \mathrm{min})$ : } \\
\hline$\leq 0.1$ & $5(8)$ & $5(8)$ \\
\hline$>0.1$ & $4(6)$ & $2(3)$ \\
\hline \multicolumn{3}{|l|}{ Clinical status on seven level ordinal scale: } \\
\hline 4: Admitted to hospital, receiving supplemental oxygen & $39(60)$ & $28(44)$ \\
\hline $\begin{array}{l}\text { 5: Admitted to hospital, receiving non-invasive ventilation or high flow } \\
\text { oxygen through nasal cannula }\end{array}$ & $15(23)$ & $26(41)$ \\
\hline 6: Admitted to hospital, receiving mechanical ventilation & $11(17)$ & $10(16)$ \\
\hline Mean (SD) SOFA score & $3.4(1.8)$ & $3.6(2.1)$ \\
\hline Respiratory support by mechanical ventilation & $11(17)$ & $10(16)$ \\
\hline \multicolumn{3}{|l|}{ Median (interquartile range) vital signs: } \\
\hline Respiratory rate (rpm) & $20(18-24)$ & $20(18-25)$ \\
\hline Peripheral oxygen saturation (\%) & $95(92-96)$ & $95(93-96)$ \\
\hline \multicolumn{3}{|l|}{ Laboratory results: } \\
\hline Median (interquartile range) $\mathrm{PaO}_{2}(\mathrm{~mm} \mathrm{Hg})$ & $83(70-105)(n=54)$ & $85(68-108)(n=57)$ \\
\hline Median (interquartile range) D dimer (nmol/L FEU) & $2.7(1.5-3.6)(n=58)$ & $2.2(1.6-3.8)(n=61)$ \\
\hline Mean (SD) C reactive protein (mg/L) & $160(104)(n=63)$ & $193(283)(n=63)$ \\
\hline Mean $(\mathrm{SD})$ ferritin $(\mu \mathrm{g} / \mathrm{L})$ & $1271(1259)(n=44)$ & $1385(1031)(n=55)$ \\
\hline Mean (SD) LDH (U/L) & $588(243)(n=60)$ & $631(335)(n=61)$ \\
\hline Mean $(\mathrm{SD})$ lactate $(\mathrm{mg} / \mathrm{dL})$ & $14.9(6.5)(n=46)$ & $15.0(7.9)(n=50)$ \\
\hline Median (interquartile range) prothrombin time (INR) & $1.1(1.0-1.2)(n=54)$ & $1.1(1.1-1.2)(n=60)$ \\
\hline Median (interquartile range) aPTT & $1.0(0.9-1.1)(n=53)$ & $1.1(1.0-1.3)(n=59)$ \\
\hline \multicolumn{3}{|l|}{ Type of in-hospital drugs: } \\
\hline Heparin & $53(81)$ & $54(84)$ \\
\hline Prophylactic & $50 / 53(94)$ & 48/54 (89) \\
\hline Therapeutic & $3 / 53(6)$ & $6 / 54(11)$ \\
\hline \multicolumn{3}{|l|}{ Corticosteroid: } \\
\hline None & $20(31)$ & $17(27)$ \\
\hline \multicolumn{3}{|l|}{ Prednisone equivalent: } \\
\hline$<0.5 \mathrm{mg} / \mathrm{kg} / \mathrm{day}$ & $14(21)$ & $13(20)$ \\
\hline$\geq 0.5$ and $<1.0 \mathrm{mg} / \mathrm{kg} /$ day & $15(23)$ & $18(28)$ \\
\hline$\geq 1 \mathrm{mg} / \mathrm{kg} /$ day & $16(25)$ & $16(25)$ \\
\hline $\begin{array}{l}\text { SOFA=sequential organ failure assessment; } \mathrm{PaO}_{2}=\text { partial pressure of oxygen; } \mathrm{FEU}= \\
\text { INR=international normalised ratio; aPTT=activated partial thrombin. } \\
\text { *Cyclosporin, mycophenolate mofetil, and tacrolimus. } \\
\text { tAntihypertensive }(n=33) \text {, hypoglycaemic agents }(n=22) \text {, statins }(n=10) \text {, thyroid ho } \\
\text { anticonvulsants }(n=1) \text {. }\end{array}$ & brinogen equivalent units; $L D H=l$ & $\begin{array}{l}\text { dehydrogenase; } \\
\text { tidepressants }(n=4) \text {, }\end{array}$ \\
\hline
\end{tabular}

Patients assigned to tocilizumab had a lower duration of hospital stay (mean 11.3 (SD 8.0) v 14.7 (8.2) days; rate ratio $0.70,95 \%$ confidence interval 0.55 to 0.87 ). Duration of hospital stay remained lower in patients assigned to tocilizumab in a post hoc analysis including only patients discharged alive (11.9 (8.4) v14.8 (8.6) days; rate ratio $0.75,0.58$ to 0.94 ). No significant differences were found in other secondary outcomes, including ventilator-free days within 29 days, time to independence from supplemental oxygen within 


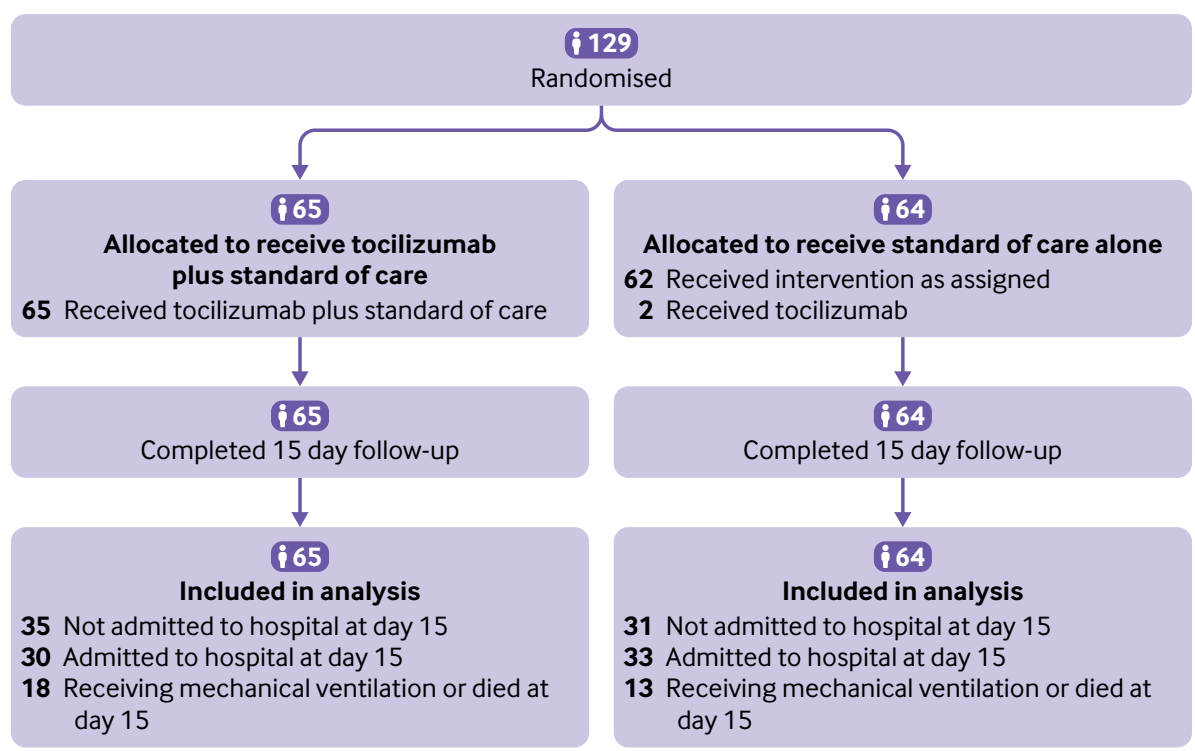

Fig 1 | Allocation, follow-up, and analysis of trial participants

29 days, secondary infections, and thromboembolic events.

Safety

A total of 29 (43\%) patients assigned to tocilizumab and 21 (34\%) assigned to standard care had adverse events $(\mathrm{P}=0.26)$ (table 3). Serious adverse events occurred in $11(16 \%)$ patients in the tocilizumab group and seven $(11 \%)$ in the standard care alone group. No detectable differences were found in the incidence of any specific adverse event between patients who received tocilizumab compared with those who did not.

\section{Inflammatory markers and cytokines}

Levels of interleukin 6 were higher among patients assigned to tocilizumab plus standard care compared with those assigned to standard care alone on days 5 and 8 (see supplementary figure S2 and table S9). Levels of $\gamma$ interferon were higher on day 5 , but not on day 8 , among patients assigned to tocilizumab. Among patients assigned to tocilizumab, interleukin 10 was not significantly different on day 5 but was higher on day 8 . C reactive protein was lower in the tocilizumab group compared with control group on both day 5 and day 8. No significant difference was found between treatment groups in the serum concentration of other cytokines.

\section{Discussion}

In this open label, multicentre, randomised, controlled trial including relatively young (mean age 57 years) patients admitted to hospital with confirmed severe or critical covid-19, the use of the interleukin 6 inhibitor tocilizumab did not result in better clinical outcomes as assessed by a seven level ordinal scale at 15 days. Mortality at 15 days was increased in the group assigned to tocilizumab. Although mortality at 15 days was not a prespecified outcome in the trial, but rather a component of the primary outcome, a detrimental effect on this end point raised concerns about safety, and the data monitoring committee therefore recommended early termination of the trial. Conversely, in both groups, deaths were attributed to covid-19 related acute respiratory failure or multiple organ dysfunction. In addition, at 29 days the effect of tocilizumab on mortality was no longer statistically significant.

Our results were unexpected given the potent antiinflammatory activity of tocilizumab in rheumatoid arthritis and CAR-T. The rapid increase in cytokines usually accompanies an increase in inflammatory markers and clinical deterioration in patients with covid-19, which is reminiscent of CAR-T. ${ }^{617-19}$ Thus, it was plausible that blocking interleukin 6 in patients with covid-19 could lessen the inflammatory response and avert some of the more dire consequences of the disease. However, clinical observation and biological plausibility are often not confirmed by randomised clinical trials. ${ }^{20-23}$

The decrease in $\mathrm{C}$ reactive protein levels after tocilizumab had been administered suggests an anti-inflammatory effect. Conversely, the increase in serum interleukin 6 levels observed in our study has been described in rheumatoid arthritis and Castleman disease after tocilizumab use. ${ }^{19}$ Tocilizumab is a humanised antibody that binds the interleukin 6 receptor inhibiting interleukin 6 signalling. In rheumatoid arthritis, despite an increase in interleukin 6 levels, inflammatory markers and clinical manifestations improve. Thus, the increase in serum interleukin 6 might represent an inhibition of interleukin 6 receptor mediated clearance and continued disease activity. ${ }^{24}$ The lack of statistically significant changes in serum interleukin 2, interleukin 4 , and tumour necrosis factor- $\alpha$ levels in patients receiving tocilizumab possibly indicates that this treatment intervention did not interfere with the 


\begin{tabular}{|c|c|c|c|c|c|}
\hline Outcomes & $\begin{array}{l}\text { Tocilizumab } \\
\text { group }(n=65)\end{array}$ & $\begin{array}{l}\text { Control group } \\
(n=64)\end{array}$ & $\begin{array}{l}\text { Effect } \\
\text { estimate }\end{array}$ & Effect size $(95 \% \mathrm{Cl})$ & Pvalue \\
\hline \multicolumn{6}{|l|}{ Primary endpoint } \\
\hline Receiving mechanical ventilation or died at day $15^{*}$ & $18(28)$ & $13(20)$ & $\begin{array}{l}\text { Odds ratio } \\
1-5 \vee 6-7\end{array}$ & 1.54 (0.66 to 3.66$)$ & 0.32 \\
\hline \multicolumn{6}{|l|}{ Clinical status (7 level ordinal scale) at day 15: } \\
\hline 1: Not admitted to hospital, no limitation on activities & $32(49)$ & $26(41)$ & & & \\
\hline 2: Not admitted to hospital, limitation on activities & $3(5)$ & $5(8)$ & & & \\
\hline 3: Admitted to hospital, not receiving supplemental oxygen & $6(9)$ & $6(9)$ & & & \\
\hline 4. Admitted to hospital, receiving supplemental oxygen & $6(9)$ & $10(16)$ & & & \\
\hline $\begin{array}{l}\text { 5. Admitted to hospital, receiving non-invasive ventilation or high } \\
\text { flow oxygen through nasal cannula }\end{array}$ & $0(0)$ & $4(6)$ & & & \\
\hline 6: Admitted to hospital, receiving mechanical ventilation & $7(11)$ & $11(17)$ & & & \\
\hline 7: Death & $11(17)$ & $2(3)$ & & & \\
\hline \multicolumn{6}{|l|}{ Secondary endpoints } \\
\hline Mortality up to 28 days & $14(21)$ & $6(9)$ & Odds ratio & $2.70(0.97$ to 8.35$)$ & 0.07 \\
\hline In-hospital mortality & $14(21)$ & $6(9)$ & Odds ratio & $2.70(0.97$ to 8.35$)$ & 0.02 \\
\hline \multicolumn{6}{|l|}{ Mean (SD) SOFA score: } \\
\hline Day 8 & $4.1(3.9)$ & $3.4(3.0)$ & Mean ratio & $1.20(0.87$ to 1.64$)$ & 0.26 \\
\hline Day 15 & $4.3(3.6)$ & $4.3(3.6)$ & Mean ratio & $0.99(0.65$ to 1.49$)$ & 0.95 \\
\hline Clinical status (6 level ordinal scale) at day 8: & & & $\begin{array}{l}\text { Odds ratio } \\
1-4 \vee 5-6\end{array}$ & 0.91 (0.44 to 1.89$)$ & 0.79 \\
\hline 1: Not admitted to hospital & $23(35)$ & $16(25)$ & & & \\
\hline 2: Admitted to hospital, not receiving supplemental oxygen & $7(11)$ & $7(11)$ & & & \\
\hline 3: Admitted to hospital, receiving supplemental oxygen & $10(15)$ & $12(19)$ & & & \\
\hline $\begin{array}{l}\text { 4: Admitted to hospital, receiving non-invasive ventilation or high } \\
\text { flow oxygen through nasal cannula }\end{array}$ & $1(1)$ & $4(6)$ & & & \\
\hline 5: Admitted to hospital, receiving mechanical ventilation & $19(29)$ & $24(37)$ & & & \\
\hline 6: Death & $5(8)$ & $1(2)$ & & & \\
\hline Clinical status (7 level ordinal scale) at day 29: & & & $\begin{array}{l}\text { Odds ratio } \\
1-5 \vee 6-7\end{array}$ & 2.17 (0.88 to 5.60$)$ & 0.10 \\
\hline 1: Not admitted to hospital, no limitation on activities & $34(52)$ & $32(50)$ & & & \\
\hline 2: Not admitted to hospital, limitation on activities & $8(12)$ & $16(25)$ & & & \\
\hline 3: Admitted to hospital, not receiving supplemental oxygen & $4(6)$ & $2(3)$ & & & \\
\hline 4: Admitted to hospital, receiving supplemental oxygen & $1(1)$ & $4(6)$ & & & \\
\hline $\begin{array}{l}\text { 5: Admitted to hospital, receiving non-invasive ventilation or high } \\
\text { flow nasal cannula }\end{array}$ & $0(0)$ & $0(0)$ & & & \\
\hline 6: Admitted to hospital, receiving mechanical ventilation & $4(6)$ & $4(6)$ & & & \\
\hline 7: Death + & $14(21)$ & $6(9)$ & & & \\
\hline Mean (SD) ventilator-free days within 29 days $¥$ & $19.4(12.0)$ & $20.5(10.8)$ & Rate ratio & 1.12 (0.86 to 1.99$)$ & 0.53 \\
\hline $\begin{array}{l}\text { Median (interquartile range) time to supplemental } \\
\text { oxygen independence within } 29 \text { days }\end{array}$ & $6(5-12)$ & $10(8-14)$ & Hazard ratio & 1.37 (0.92 to 2.04) & 0.12 \\
\hline Mean (SD) duration of hospital stay (days) & $11.3(8.0)$ & $14.7(8.2)$ & Rate ratio & $0.70(0.55$ to 0.87$)$ & 0.001 \\
\hline Secondary infections§ & $10(15)$ & $10(16)$ & Odds ratio & $0.99(0.37$ to 2.67$)$ & 0.98 \\
\hline Thromboembolic events & $3(5)$ & $4(6)$ & Odds ratio & $0.72(0.14$ to 3.40$)$ & 0.67 \\
\hline \multicolumn{6}{|c|}{$\begin{array}{l}\text { SOFA=sequential organ failure assessment. } \\
\text { *Primary outcome, clinical status measured at } 15 \text { days using seven level ordinal scale, was analysed as a composite of death or mechanical ventilation as prespecified in the statistical analysis } \\
\text { plan because the assumption of proportional odds, necessary to analyse the original seven level ordinal scale, did not hold. } P=0.32 \text { for primary outcome analysis. } \\
\text { tDeath before day } 29 ; \text { ventilator-free days considered } 0 \text {. } \\
\text { t19 deaths were associated with covid- } 19 \text { related acute respiratory failure or multiple organ dysfunction and one death with covid-19 related cerebral haemorrhage. } \\
\text { \$Tocilizumab group: bloodstream }(n=5) \text {, respiratory }(n=5) \text {, skin soft issue }(n=1) \text {. One patient had a respiratory and bloodstream infection. Control group: bloodstream ( } n=3) \text {, respiratory }(n=7) \text {, } \\
\text { indeterminate }(n=1) \text {, bacteraemia }(n=1) \text {. One patient had a respiratory infection and bacteraemia. } \\
\text { qThromboembolic events included stroke, myocardial infarction, deep vein thrombosis, and pulmonary embolism. Tocilizumab group: deep venous thrombosis }(n=2) \text { and stroke }(n=1) \text {. Control } \\
\text { group: pulmonary embolism }(n=3) \text { and stroke }(n=1) \text {. }\end{array}$} \\
\hline
\end{tabular}

core events in SARS-CoV-2 infection, but rather is restricted to the modulation of downstream effects of interleukin $6 .^{24}$

These results raise questions about an antiinflammatory approach in the treatment of covid-19 beyond corticosteroids, which might also have an immune modulation role in covid-19. Dexamethasone was found to be associated with a reduction in 28 day mortality in patients with covid-19 who needed supplemental oxygen or mechanical ventilation. ${ }^{25}$ No other anti-inflammatory agents have been shown to be beneficial in covid-19. A single anticytokine approach might not inhibit the breadth of the inflammatory response in covid-19 and could have a limited role as a single agent.

\section{Comparison with other studies}

Non-randomised studies have suggested that tocilizumab might have a role in controlling cytokine release syndrome associated with covid-19. ${ }^{26-34}$ Most observational studies, although not all, suggest a benefit of tocilizumab on clinical outcomes. ${ }^{26} 303133$ Results from observational studies are, however, limited owing to a high risk of confounding. Conversely, randomised controlled trials have not shown a beneficial effect of tocilizumab on their respective 

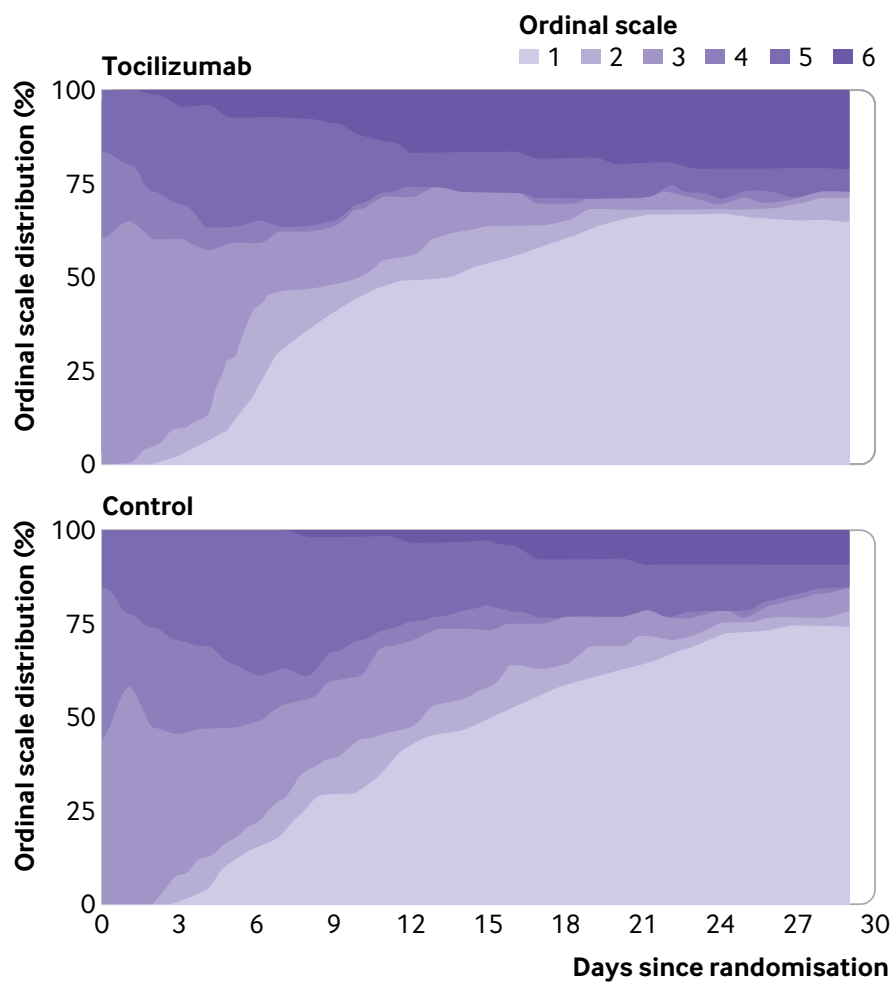

Fig 2 | Relative distribution of patient status over time stratified by treatment group. Six level ordinal scale-1: not admitted to hospital; 2: admitted to hospital, not receiving supplemental oxygen; 3: admitted to hospital, receiving supplemental oxygen; 4: admitted to hospital, receiving non-invasive ventilation or high flow oxygen through a nasal cannula; 5: receiving mechanical ventilation; 6: death

primary outcomes..$^{20-23}$ In the Study to Evaluate the Safety and Efficacy of Tocilizumab in Patients With Severe COVID-19 Pneumonia (COVACTA), enrolling 452 hypoxaemic patients, tocilizumab had no effect on clinical outcomes at 28 days, assessed with the seven level ordinal scale. ${ }^{20}$ Length of intensive care unit and hospital stay, however, were lower among patients assigned to tocilizumab. The CORIMUNO trial included 131 hypoxaemic patients and did not show an effect of tocilizumab on clinical outcomes at 28 days. ${ }^{21}$ The Boston Area COVID-19 Consortium (BACC) Bay Tocilizumab Trial randomised 242 hypoxaemic patients and reported no difference in time to intubation or death during 28 days. ${ }^{22}$ Another study, which enrolled 126 hypoxaemic patients, reported no benefit on disease progression at day $14 .^{23}$

Identifying a population that would likely benefit from tocilizumab has not been possible so far. We found no benefit of tocilizumab among patients with a more pronounced inflammatory phenotype and concurrent lung damage, defined by an increase of at least two laboratory markers and hypoxaemia. Observational studies have suggested that a beneficial effect of tocilizumab is related to starting treatment early in the course of illness. ${ }^{35-38}$ For example, in the STOP-covid trial $^{35}$ tocilizumab was associated with decreased mortality only among patients with a time from onset of symptoms to intensive care unit admission equal to or lower than three days. Conversely, in all randomised trials assessing tocilizumab, including our trial, average duration of symptoms at baseline ranged from 8 to 12 days. ${ }^{20-23}$ Nevertheless, in a post hoc subgroup analysis, we found no evidence that earlier $(\leq 10$ days) versus later ( $>10$ days) initiation of tocilizumab modifies the treatment effect of tocilizumab on clinical outcomes (see supplementary table S10).

\section{Limitations of this study}

Our baseline data were well balanced, except for respiratory support and use of azithromycin. The level of respiratory support, an important prognostic marker, was lower among patients assigned to tocilizumab.

\begin{tabular}{|c|c|c|c|}
\hline Events & Tocilizumab group $(n=67)$ & Control group $(n=62)$ & $P$ value \\
\hline Any adverse events & $29(43)$ & $21(34)$ & 0.26 \\
\hline \multicolumn{4}{|c|}{ Reported severe adverse event, according to classification*: } \\
\hline Any severe adverse event & $11(16)$ & $7(11)$ & 0.45 \\
\hline Raised ALT, AST, or bilirubin level & $7(10)$ & $3(5)$ & 0.33 \\
\hline Anaemia & $3(4)$ & $3(5)$ & 1.00 \\
\hline Pneumothorax & $0(0)$ & $1(2)$ & 0.48 \\
\hline Neutropenia & $1(1)$ & $0(0)$ & 1.00 \\
\hline Bleeding & $1(1)$ & $0(0)$ & 1.00 \\
\hline Intracranial bleeding & $0(0)$ & $1(2)$ & 1.00 \\
\hline Sudden cardiorespiratory collapse & $4(6)$ & $1(2)$ & 0.37 \\
\hline \multicolumn{4}{|l|}{ Non-severe adverse events: } \\
\hline Any non-severe adverse event & $24(36)$ & $15(24)$ & 0.18 \\
\hline Raised ALT, AST, or bilirubin level & $11(16)$ & $4(6)$ & 0.10 \\
\hline Anaemia & $7(10)$ & $10(16)$ & 0.44 \\
\hline Haemorrhage & $1(1)$ & $1(2)$ & 1.00 \\
\hline Neutropenia & $1(1)$ & $0(0)$ & 1.00 \\
\hline Thrombocytopenia & $4(6)$ & $0(0)$ & 0.12 \\
\hline Neutrophilia & $1(1)$ & $0(0)$ & 1.00 \\
\hline Anxiety & $1(1)$ & $0(0)$ & 1.00 \\
\hline Lymphopenia & $0(0.0)$ & $1(2)$ & 1.00 \\
\hline Atrial fibrillation & $1(1)$ & $0(0)$ & 1.00 \\
\hline Hypoacusis & $1(1)$ & $0(0)$ & 1.00 \\
\hline
\end{tabular}


The results of a post hoc analysis adjusted for baseline levels of respiratory support were consistent with those of the main analysis and did not show a significant effect on the primary outcome. Although baseline use of azithromycin was less common among patients assigned to tocilizumab, the drug has proven to be ineffective for patients admitted to hospital with covid-19. ${ }^{39-41}$

Our trial has other limitations. Firstly, it was an open label trial. Although assessment of the primary outcome, a seven level ordinal scale at 15 days, is objective, the decision to perform tracheal intubation and its timing depends on the operator. Secondly, the sample size was relatively small. Thus the $95 \%$ confidence interval around the effect on the primary outcome was wide, compatible with odds ratios between 0.66 (benefit of tocilizumab) and 3.66 (harm with tocilizumab). Thirdly, the distribution of the seven level ordinal scale at 15 days was not compatible with proportional odds assumptions, which required reclassification of the outcome as a binary variable. This finding led to a further reduction in the statistical power to detect a treatment effect on the primary outcome. Fourthly, we did not record the number of patients assessed for eligibility. Fifthly, after randomisation we collected information on concomitant treatment into broad classes: antivirals, corticosteroids, and antibiotics. These co-interventions were administered similarly for patients assigned to both treatment groups up to day 15. However, we are unable to report on use of these drugs according to specific agents. In any case, except for corticosteroids, and possibly remdesivir (not available in Brazil), no antiviral or antibiotic has been shown to modify the clinical course of patients admitted to hospital with covid-19. ${ }^{39-41}$

\section{Conclusions}

In this trial including patients admitted to hospital with severe or critical covid-19, the use of tocilizumab plus standard care was not superior to standard care alone in improving patients' clinical status at 15 days, and might have increased mortality.

\footnotetext{
AUTHOR AFFILIATIONS

${ }^{1}$ BP-A Beneficência Portuguesa de São Paulo, Rua Maestro Cardim, 769-Bela Vista, São Paulo-SP, 01323-001, Brazil

${ }^{2}$ Brazilian Research in Intensive Care Network-BRICNet, São Paulo, Brazil

${ }^{3}$ Hospital Moinhos de Vento, Porto Alegre, Brazil

${ }^{4} \mathrm{HCor}$ Research Institute, São Paulo, Brazil

${ }^{5}$ Anesthesiology, Pain and Intensive Care Department, Universidade Federal de São Paulo, São Paulo, Brazil

${ }^{6}$ Duke Clinical Research Institute, Duke University Medical Center, Durham, NC, USA

${ }^{7}$ Brazilian Clinical Research Institute, São Paulo, Brazil

${ }^{8}$ Academic Research Organization, Hospital Israelita Albert Einstein, São Paulo, Brazil

${ }^{9}$ Hospital Sírio-Libanês, São Paulo, Brazil

${ }^{10}$ International Research Center-Hospital Alemão Oswaldo Cruz, São Paulo, Brazil

${ }^{11}$ Fleury Medicine and Health Laboratories, Grupo Fleury, São Paulo, Brazil

${ }^{12}$ Santa Casa de Porto Alegre, Porto Alegre, Brazil
}

${ }^{13}$ Hospital Santa Paula, São Paulo, Brazil

${ }^{14}$ Hospital SEPACO, São Paulo, Brazil

${ }^{15}$ Hospital Alemão Oswaldo Cruz, São Paulo, Brazil

${ }^{16}$ Estátikos Consultoria Estatística, São Paulo, Brazil

The collaborators are listed in the supplementary file. The trial was designed by the executive committee (see supplementary file). The executive committee vouches for the completeness and accuracy of the data and for the fidelity of the trial to the protocol.

Contributors: ABC, DLCF, JP, PS, and VCV conceived the study, wrote the protocol, recruited patients, and drafted the manuscript. $A B C, R G R$, FRM, FGZ, OB, LCPA, RDL, AA, LKD, and CGC developed the protocol and approved the final version for the Coalition covid-19 Brazil Group, recruited patients, participated in interim discussions, and reviewed the manuscript. CZO developed the REDCap database and attended to all data collection related issues. LPD and LMI performed the statistical analysis. LECA, AFS, and MCP coordinated exploratory sample collection and will be performing the exploratory analysis, participated in the protocol development an interim discussion and reviewed the manuscript. VCV, ABC, DLCF, JP, and PS act as guarantors, accept full responsibility for the work, had access to the data, and controlled the decision to publish. The corresponding author attests that all listed authors meet authorship criteria and that no others meeting the criteria have been omitted.

Funding: This trial was funded by the hospitals and research institutes participating in the Coalition covid-19 Brazil. The exploratory laboratory analysis was conducted and funded by Fleury Laboratory in São Paulo, Brazil. Instituto Votorantim provided a donation for the purchase of tocilizumab for this study. It had no role in the design of the trial, the conduct, the analysis, or the decision to submit the manuscript for publication.

Competing interests: All authors have completed the ICMJE uniform disclosure form at www.icmje.org/coi_disclosure.pdf and declare: support from hospitals and research institutes participating in the Coalition covid-19 Brazil, Fleury Laboratory in São Paulo, Brazil, and Instituto Votorantim for the submitted work. JAGGP reports support from Pfizer, Jansen, Sanofi, United Medical, MSD, Astellas, Astra Zeneca, and Eurofarma. DLCF has received grants from Abbvie, AstraZeneca, Bristol Myers Squibb, Boehringer Ingelheim, Celltrion, Daichii Sankyo, GSK, Janssen, Takeda, Novartis, Pfizer, Sandoz, Sanofi, Viracta, Onconova, AGIOS, Astellas, MEDAC, Roche, Janssen, ABBVIE, Novartis, Takeda, Amgen, Libbs, Pfizer, Bristol Myers Squibb, Celgene, and Eurofarma. LKD receives a research grant from Bristol Myers Squibb, Roche, and Boehringer Ingelheim. RDL received grants and personal fees from Bristol Myers Squibb, Pfizer, Boehringer Ingelheim, and Bayer, and grants from Amgen, GlaxoSmithKline, Medtronic, and Sanofi Aventis outside the submitted work. OB received grants from AstraZeneca, Bayer, Pfizer, Novartis, Amgen, Boehringer Ingelheim, and Servier. DJBM reports support from Novartis. ANC reports receiving grants from Pfizer. AA reports receiving grants from Sanofi-Pasteur, Bayer, and Population Health Research Institute, and personal fees from Bayer, Boehringer Ingelheim, Novo Nordisk, and Lilly. ABC reports grants from Bactiguard, Ionis Pharmaceuticals, Brazilian Ministry of Health (PROADI-SUS), Brazilian Ministry of Science and Technology, Bayer, Pfizer, Hillrom, Fisher \& Paykel, and Baxter. PS received grants from Roche, BioCryst, Amgen, Merck, Eurofarma, Novartis, Abbvie, Janssen, Alexion-Advisory, Novartis, Abbvie, Janssen, and Alexion.

Ethical approval: This trial was approved by the Brazilian National Commission for Research Ethics and the ethics committees at all participating sites.

Data sharing: Deidentified participant data and a dictionary of variables will be available to clinical researchers three months after publication on request to the corresponding author at viviane.veiga@ bp.org.br. The Coalition covid-19 Brazil executive committee will oversee and decide about the use of the study data upon receiving a reasonable request. The executive committee reviewed and commented on any draft manuscripts before publication.

The lead author (VCV) affirms that the manuscript is an honest, accurate, and transparent account of the study being reported; that no important aspects of the study have been omitted; and that any discrepancies from the study as planned (and, if relevant, registered) have been explained.

Dissemination to participants and related patient and public communities: There are no plans to disseminate the results of the research to study participants. Study results will be shared with the public through press release, social media, conference presentations, and https://www.bp.org.br. 
Provenance and peer review: Not commissioned; externally peer reviewed.

This is an Open Access article distributed in accordance with the Creative Commons Attribution Non Commercial (CC BY-NC 4.0) license, which permits others to distribute, remix, adapt, build upon this work non-commercially, and license their derivative works on different terms, provided the original work is properly cited and the use is noncommercial. See: http://creativecommons.org/licenses/by-nc/4.0/.

1 WHO Coronavirus Disease (COVID-19) Dashboard. World Health Organization, 2020. https://Covid19.who.int/. [Accessed 19 October 2020.]

2 Jones L, Palumbo D, Brown D. Coronavirus: A visual guide to the economic impact. BBC News: BBC; 2020

3 Nicola M, Alsafi Z, Sohrabi C, et al. The socio-economic implications of the coronavirus pandemic (COVID-19): A review. Int Surg 2020;78:185-93. doi:10.1016/j.ijsu.2020.04.018

4 Wu Z, McGoogan JM. Characteristics of and important lessons from the coronavirus disease 2019 (COVID-19) outbreak in China: Summary of a report of 72,314 cases from the Chinese Center for Disease Control and Prevention. JAMA 2020;323:1239-42. doi:10.1001/jama.2020.2648

5 Merad M, Martin JC. Pathological inflammation in patients with COVID-19: a key role for monocytes and macrophages. Nat Rev Immunol 2020;20:355-62. doi:10.1038/s41577-020-0331-4

6 Moore JB, June $\mathrm{CH}$. Cytokine release syndrome in severe COVID-19. Science 2020;368:473-4. doi:10.1126/science.abb8925

7 Blanco-Melo D, Nilsson-Payant BE, Liu WC, et al. Imbalanced host response to SARS-CoV-2 drives development of COVID-19. Cell 2020;181:1036-1045.e9. doi:10.1016/j.cell.2020.04.026

8 Chen G, Wu D, Guo W, et al. Clinical and immunological features of severe and moderate coronavirus disease 2019. J Clin Invest 2020;130:2620-9. doi:10.1172/JCl137244

9 Scott LJ. Tocilizumab: A review in rheumatoid arthritis. Drugs 2017;77:1865-79. doi:10.1007/s40265-017-0829-7

10 Tanaka T, Narazaki M, Kishimoto T. IL-6 in inflammation, immunity, and disease. Cold Spring Harb Perspect Biol 2014;6:a016295. doi:10.1101/cshperspect.a016295

11 Rose-John S, Winthrop K, Calabrese L. The role of IL-6 in host defence against infections: immunobiology and clinical implications. Nat Rev Rheumatol 2017;13:399-409. doi:10.1038/nrrheum.2017.83

12 Herold T, Jurinovic V, Arnreich C, et al. Elevated levels of IL-6 and CRP predict the need for mechanical ventilation in COVID-19. I Allergy Clin Immunol 2020;146:128-136.e4. doi:10.1016/j.jaci.2020.05.008

13 Laguna-Goya R, Utrero-Rico A, Talayero P, et al. IL-6-based mortality risk model for hospitalized patients with COVID-19. J Allergy Clin Immunol 2020;146:799-807.e9. doi:10.1016/j.jaci.2020.07.009

14 Farias DLC, Prats J, Cavalcanti AB, et al. Rationale and design of tocilizumab in patients with moderate to severe COVID-19: an open label multicentre randomised controlled trial (TOCIBRAS). Revista Brasileira de terapia intensiva 2020. Rev Bras Ter Intensiva 2020;32:337-47. doi:10.5935/0103-507X.20200060

15 Siemieniuk R, Rochwerg B, Agoritsas T, et al. A living WHO guideline on drugs for covid-19. BMJ 2020;370:m3379. doi:10.1136/bmj.m3379

16 R Core Team. $R$ : a language and environmnet for statistical computing. R Foundation for Statistical Computing, 2020.

17 Mehta P, McAuley DF, Brown M, Sanchez E, Tattersall RS, Manson JJ, HLH Across Speciality Collaboration, UK. COVID-19: consider cytokine storm syndromes and immunosuppression. Lancet 2020;395:10334. doi:10.1016/S0140-6736(20)30628-0

18 Vaninov N. In the eye of the COVID-19 cytokine storm. Nat Rev Immunol 2020;20:277. doi:10.1038/s41577-020-0305-6

19 Quartuccio L, Sonaglia A, Pecori D, et al. Higher levels of IL-6 early after tocilizumab distinguish survivors from nonsurvivors in COVID-19 pneumonia: A possible indication for deeper targeting of IL-6. J Med Virol 2020. doi:10.1002/jmv.26149

20 Rosas I, Brau N, Waters M, et al. Tocilizumab in hospitalized patients with COVID-19 pneumonia. medRxiv 2020.08.27.20183442. https://doi.org/10.1101/2020.08.27.20183442

21 Hermine O, Mariette X, Tharaux PL, et al. Effect of Tocilizumab vs usual care in adults hospitalized with COVID-19 and moderate or severe pneumonia: a randomized clinical trial. JAMA Intern Med 2021;181:32-40. doi:10.1001/jamainternmed.2020.6820

22 Stone JH, Frigault MJ, Serling-Boyd NJ, et al. Efficacy of tocilizumab in patients hospitalized with COVID-19. N Engl / Med 2020;383:233334. doi:10.1056/NEIMoa2028836

23 Salvarani C, Dolci G, Massari M, et al, RCT-TCZ-COVID-19 Study Group. Effect of Tocilizumab vs Standard Care on Clinical Worsening in Patients Hospitalized With COVID-19 Pneumonia: A Randomized Clinical Trial. JAMA Intern Med 2021:181:24-31. doi:10.1001/ jamainternmed.2020.6615
24 Nishimoto N, Terao K, Mima T, Nakahara H, Takagi N, Kakehi T. Mechanisms and pathologic significances in increase in serum interleukin-6 (IL-6) and soluble IL-6 receptor after administration of an anti-IL-6 receptor antibody, tocilizumab, in patients with rheumatoid arthritis and Castleman disease. Blood 2008;112:395964. doi:10.1182/blood-2008-05-155846

25 Horby P, Lim WS, Emberson JR, et al. Dexamethasone in Hospitalised Patients with Covid-19 - Preliminary Report. N Engl J Med 2020. doi:10.1056/NEJMoa2021436

26 Somers EC, Eschenauer GA, Troost JP, et al. Tocilizumab for treatment of mechanically ventilated patients with COVID-19. Clin Infect Dis 2020. doi:10.1093/cid/ciaa954

27 Tomasiewicz K, Piekarska A, Stempkowska-Rejek J, et al. Tocilizumab for patients with severe COVID-19: a retrospective, multi-center study. Expert Rev Anti Infect Ther 2020. doi:10.1080/14787210.20 20.1800453

28 Campochiaro C, Della-Torre E, Cavalli G, et al, TOCl-RAF Study Group. Efficacy and safety of tocilizumab in severe COVID-19 patients: a single-centre retrospective cohort study. Eur J Intern Med 2020:76:43-9. doi:10.1016/j.ejim.2020.05.021

29 Kewan T, Covut F, Al-Jaghbeer MJ, Rose L, Gopalakrishna KV, Akbik B. Tocilizumab for treatment of patients with severe COVID-19: A retrospective cohort study. EClinicalMedicine 2020;24:100418. doi:10.1016/j.eclinm.2020.100418

30 Rossotti R, Travi G, Ughi N, et al, Niguarda COVID-19 Working Group. Safety and efficacy of anti-il6-receptor tocilizumab use in severe and critical patients affected by coronavirus disease 2019 A comparative analysis. J Infect 2020;81:e11-17. doi:10.1016/j. jinf.2020.07.008

31 Moreno-Pérez O, Andres M, Leon-Ramirez JM, et al. Experience with tocilizumab in severe COVID-19 pneumonia after 80 days of followup: A retrospective cohort study. J Autoimmun 2020;114:102523. doi:10.1016/j.jaut2020.102523

32 Colaneri M, Bogliolo L, Valsecchi P, et al. The Covid Irccs San Matteo Pavia Task Force. Tocilizumab for Treatment of Severe COVID-19 Patients: Preliminary Results from SMAtteo COvid19 REgistry (SMACORE). Microorganisms 2020;8:695. doi:10.3390/ microorganisms 8050695

33 Klopfenstein T, Zayet S, Lohse A, et al, HNF Hospital Tocilizumab multidisciplinary team. Tocilizumab therapy reduced intensive care unit admissions and/or mortality in COVID-19 patients. Med Mal Infect 2020;50:397-400. doi:10.1016/j.medmal.2020.05.001

34 Canziani LM, Trovati S, Brunetta E, et al, Humanitas and Gavazzeni / Castelli COVID-19 Task Forces. Interleukin-6 receptor blocking with intravenous tocilizumab in COVID-19 severe acute respiratory distress syndrome: A retrospective case-control survival analysis of 128 patients. J Autoimmun 2020;114:102511. doi:10.1016/j. jaut.2020.102511

35 Gupta S, Wang W, Hayek SS, et al, STOP-COVID Investigators. Association between early treatment with tocilizumab and mortality among critically ill patients with COVID-19. JAMA Intern Med 2021;181:41-51. doi:10.1001/jamainternmed.2020.6252

36 Petrak RM, Van Hise NW, Skorodin NC, et al. Early tocilizumab dosing is associated with improved survival in critically ill patients infected with SARS-COV-2. medRxiv 2020.10.27.20211433; doi:10.1101/2020.10.27.20211433

37 Capra R, De Rossi N, Mattioli F, et al. Impact of low dose tocilizumab on mortality rate in patients with COVID-19 related pneumonia. Eur $)$ Intern Med 2020;76:31-5. doi:10.1016/j.ejim.2020.05.009

38 Langer-Gould A, Smith JB, Gonzales EG, et al. Early identification of COVID-19 cytokine storm and treatment with anakinra or tocilizumab. Int J Infect Dis 2020;99:291-7. doi:10.1016/j.ijid.2020.07.081

39 Furtado RHM, Berwanger O, Fonseca HA, et al, COALITION COVID-19 Brazil II Investigators. Azithromycin in addition to standard of care versus standard of care alone in the treatment of patients admitted to the hospital with severe COVID-19 in Brazil (COALITION II): a randomised clinical trial. Lancet 2020;396:959-67. doi:10.1016/ S0140-6736(20)31862-6

40 Cavalcanti AB, Zampieri FG, Rosa RG, et al, Coalition Covid-19 Brazil Investigators. Hydroxychloroquine with or without Azithromycin in Mild-to-Moderate Covid-19. N Engl J Med 2020;383:2041-52. doi:10.1056/NEJMoa2019014.

41 Horby PW, Roddick A, Spata E, et al. Azithromycin in hospitalized patients with covid-19 (RECOVERY): a randomized, controlled, openlabel, platform trial. medRxiv 2020.12. 10.20245944. https://doi.or g/10.1101/2020.12.10.20245944

Supplementary information: additional information, tables S1-S10, and figures S1 and S2 